\title{
Cirurgia não Ginecológica em Pacientes com Câncer de Ovário
}

\author{
Nongynecologic Surgery in Patients with Ovarian Cancer
}

\begin{abstract}
Agnaldo Lopes da Silva-Filho ${ }^{1}$, Eduardo Batista Cândido ${ }^{1}$, Maurício Bechara Noviello ${ }^{1}$, Admário Silva Santos-Filho ${ }^{1}$, Paulo Traiman ${ }^{3}$, Sérgio Augusto Triginelli ${ }^{1}$, José Renan Cunha-Melo
\end{abstract}

\section{RESUMO}

Objetivo: avaliar a freqüência da utilização de procedimentos cirúrgicos não ginecológicos no tratamento do câncer de ovário, assim como descrever as suas complicações.

Métodos: foram incluidas retrospectivamente 82 pacientes, atendidas no periodo de fevereiro de 1999 a outubro de 2003, e que haviam sido submetidas a laparotomia para tratamento cirúrgico do câncer de ovário. Foram incluidas no estudo apenas pacientes com

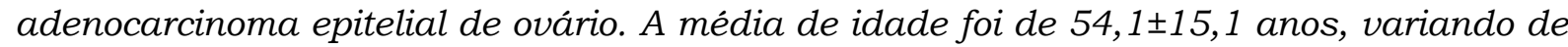
22 a 89 anos. O estadiamento (FIGO) mostrou 5 pacientes no estádio I (6,1\%), 18 pacientes no II $(21,9 \%), 40$ pacientes no III $(48,8 \%)$ e 19 pacientes no IV $(23,2 \%)$. As pacientes foram divididas em 2 grupos: aquelas em que foram realizados exclusivamente procedimentos ginecológicos e aquelas com realização de procedimentos não ginecológicos. As diferenças entre as médias foram analisadas pelo teste $t$ de Student. As comparações entre grupos independentes foram feitas pelo teste do $\chi^{2}$.

Resultados: em 35 pacientes (42,7\%) foram realizados procedimentos cirúrgicos não ginecológicos, incluindo: 17 colostomias, 16 enterectomias, oito peritonectomias pélvicas, sete colectomias, cinco ressecções parciais de cúpula frênica, quatro cistectomias parciais, quatro esplenectomias, duas ileostomias e uma hepatectomia parcial. Todas as pacientes submetidas a procedimentos não ginecológicos apresentavam-se nos estádios III e IV. Esse

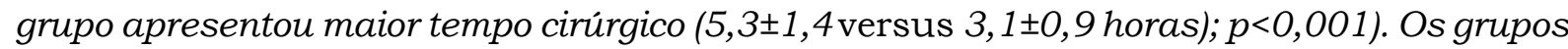
não mostraram diferenças significativas em relação à necessidade de hemotransfusão (42,2 versus 40\%; $p=0,512)$ e tempo de internação $(11,5 \pm 7,2$ versus $10 \pm 9,9$ dias; $p=0,454)$. A realização de procedimentos cirúrgicos não ginecológicos associou-se a maior taxa de complicações pós-operatórias 37\% versus $(17,1 \% ; p=0,042)$, sendo que duas pacientes desse grupo $(2,4 \%)$ evoluiram para o óbito.

Conclusões: os procedimentos cirúrgicos não ginecológicos são freqüentemente empregados no tratamento de pacientes com câncer de ovário. A realização dessas cirurgias está associada a maior tempo cirúrgico e maior taxa de complicações pós-operatórias.

PALAVRAS-CHAVE: Câncer de ovário. Citorredução. Complicações da cirurgia.

Departamentos de Ginecologia e Obstetrícia ${ }^{1}$ e Cirurgia $^{2}$ da Faculdade de Medicina da Universidade Federal de Minas Gerais.

Departamento de Ginecologia e Obstetrícia da Faculdade de Medicina de Botucatu da Universidade Estadual Paulista ${ }^{3}$.

Correspondência:

Agnaldo Lopes da Silva Filho

Avenida Pasteur 89/1310

Bairro Santa Efigênia

30150290 - Belo Horizonte - MG

Telefone: (31) 3236-6103. Fax: (31) 3296-5490

e-mail: agsilvaf@terra.com.br
Introdução

O câncer de ovário é a oitava neoplasia maligna mais diagnosticada em mulheres no Brasil e a quinta causa de morte por câncer nas mulheres americanas ${ }^{1}$. Corresponde a $1,8 \%$ dos cânceres ginecológicos e apresenta a mais elevada mortalidade entre eles ${ }^{2}$. A neoplasia maligna de ovário representa grande desafio, pois apesar dos avanços da terapia oncológica, a sobrevida das pacientes não se alterou nas últimas décadas ${ }^{1,2}$. 
O estadiamento no momento do diagnóstico tem importante valor prognóstico ${ }^{3,4}$. Cerca de $60 \%$ das pacientes são diagnosticadas nos estádios III e IV, com pior prognóstico e expectativa de vida em cinco anos de apenas 10 a $20 \%{ }^{3}$. Outro fator prognóstico, que pode ser influenciado diretamente pelo cirurgião, é a extensão da doença residual após a cirurgia primária ${ }^{5}$.

A citorredução, descrita por Meigs ${ }^{6}$ em 1935, constitui um dos princípios do tratamento cirúrgico do câncer de ovário. Pacientes submetidas à excisão completa do tumor apresentam maior sobrevida em relação àquelas cuja massa tumoral foi retirada parcialmente ${ }^{7}$. Considera-se uma citorredução satisfatória quando persiste doença residual com diâmetro inferior a um centímetro ${ }^{8-}$ ${ }^{10}$. Pacientes submetidas a uma citorredução ótima apresentam sobrevida global média de 35 meses, ao passo que aquelas com doença residual maior que um centímetro sobrevivem, em média, 18 meses $^{11}$. Uma metanálise, envolvendo 81 estudos e 6885 pacientes, mostrou a citorredução satisfatória como determinante da sobrevida em pacientes com carcinoma de ovário nos estádios III ou IV, mesmo após controle de outros fatores prognósticos ${ }^{12}$.

A disseminação do câncer de ovário na superfície peritoneal não respeita os limites anatômicos dos órgãos e vísceras abdominais e pélvicas ${ }^{13}$. Essa característica biológica implica a necessidade de se realizarem procedimentos cirúrgicos em alças intestinais, bexiga, reto e estruturas extrapélvicas, como baço, figado e diafragma, para a obtenção de uma citorredução satisfatória ${ }^{14}$. As cirurgias do trato gastrintestinal também podem ser empregadas em procedimentos paliativos e no reparo de lesões ocorridas durante a citorredução $^{15}$. Dessa forma, é fundamental que o cirurgião que se propõe a tratar o câncer de ovário tenha domínio técnico de diversos procedimentos cirúrgicos, que freqüentemente extrapolam a cirurgia ginecológica propriamente dita ${ }^{15}$. A realização desses procedimentos associa-se a um aumento da morbidade, restringindo as suas indicações para os casos com possibilidade de citorredução ótima ${ }^{8,9}$. Esse estudo tem como objetivo avaliar a freqüência da utilização de procedimentos cirúrgicos não ginecológicos no tratamento do câncer de ovário, assim como descrever as suas complicações.

\section{Pacientes e Métodos}

Foram incluídas retrospectivamente 82 pacientes, atendidas no período de fevereiro de 1999 a outubro de 2003, submetidas a laparotomia para tratamento cirúrgico do câncer de ovário. A média de idade foi de 54,1 $\pm 15,1$ anos, variando de 22 a 89 anos. Todos os procedimentos cirúrgicos foram feitos pela equipe de cirurgia pélvica do Hospital das Clínicas da UFMG. Esse estudo foi aprovado pela Comissão de Ética em Pesquisa da referida instituição.

A laparotomia em estudo constituiu o tratamento primário em 51 pacientes $(62,2 \%)$. Quimioterapia prévia já havia sido realizada em 22 pacientes $(26,8 \%)$. Das 25 pacientes $(30,5 \%)$ que haviam sido operadas previamente, $19(23,2 \%)$ foram submetidas a histerectomia total e anexectomia bilateral e $6(7,3 \%)$ foram submetidas à ooforectomia.

Foram incluídas no estudo apenas pacientes com adenocarcinoma epitelial de ovário. As pacientes foram estadiadas conforme as orientações da Federação Internacional de Ginecologia e Obstetrícia (FIGO), por laparotomia mediana, histerectomia total com anexectomia bilateral, omentectomia, biópsias peritoneais nos locais suspeitos, citologia oncótica do líquido peritoneal e linfadenectomia retroperitoneal, quando indicadas ${ }^{16}$.

As pacientes foram divididas em dois grupos: aquelas em que foram realizados exclusivamente procedimentos ginecológicos e aquelas com realização de procedimentos não ginecológicos.

Foram considerados procedimentos cirúrgicos ginecológicos: laparotomia para biópsia, ooforectomia, histerectomia, omentectomia e linfadenectomia retroperitoneal. Não ginecológicos foram considerados os procedimentos que, antes da existência de uma equipe de cirurgia pélvica, usualmente necessitavam da participação do cirurgião geral, coloproctologista ou urologista, para a sua realização. Dessa forma, foram considerados procedimentos cirúrgicos não ginecológicos: enterectomias, ressecções de cúpula frênica, esplenectomias, hepatectomias, colectomias, colostomias, ileostomias, peritonectomias pélvicas e cistectomias. A apendicectomia e colecistectomia não foram consideradas procedimentos não ginecológicos, por nem sempre serem indicadas para o tratamento específico do câncer de ovário.

As fichas preenchidas foram revisadas, sendo as informações codificadas e digitadas em banco de dados no programa SPSS para Windows versão 10.0 (SPSS Inc., Chicago, IL, USA). Esse banco de dados foi empregado também para o desenvolvimento dos cálculos estatísticos. A idade foi descrita por meio de sua média, valor mínimo, máximo e desvio padrão. As análises das diferenças entre as médias foram feitas pelo teste $t$ de 
Student. As comparações entre grupos independentes foram feitas pelo teste do $\chi^{2}$. As diferenças ou correlações com valor de $\mathrm{p}<0,05$ foram consideradas significativas.

\section{Resultados}

Segundo o estadiamento (FIGO), 5 pacientes apresentavam-se no estádio I $(6,1 \%), 18$ pacientes no estádio II $(21,9 \%), 40$ pacientes no estádio III $(48,8 \%)$ e 19 pacientes no estádio IV $(23,2 \%)$. O tempo cirúrgico médio foi de $4,3 \pm 1,6$ horas, variando de 1 a 9 horas. Houve necessidade de hemotransfusão em 33 pacientes $(40,2 \%)$. O tempo de internação hospitalar variou de 3 a 37 dias $(10,9 \pm 8,4$ dias). Complicações pós-operatórias foram observadas em 20 pacientes $(24,4 \%)$, estando as principais descritas na Tabela 1. Duas pacientes $(2,4 \%)$ evoluíram para o óbito devido à embolia pulmonar.

Tabela 1 - Complicações pós-operatórias nas pacientes submetidas a procedimentos cirúrgicos ginecológicos, pacientes submetidas a procedimentos cirúrgicos não ginecológicos e na totalidade dos casos

\begin{tabular}{lccc}
\hline $\begin{array}{l}\text { Complicações } \\
\text { pós-operatórias }\end{array}$ & $\begin{array}{c}\text { Procedimentos } \\
\text { ginecológicos }\end{array}$ & $\begin{array}{c}\text { Procedimentos } \\
\text { não }\end{array}$ & $\begin{array}{c}\text { Total de } \\
\text { ginecológicos }\end{array}$ \\
pacientes \\
Infecção do trato urinário & 8 & 4 & $\mathbf{n}$ \\
Infecção do sítio cirúrgico & 4 & 4 & 12 \\
Embolia pulmonar & 2 & 6 & 8 \\
Abscesso pélvico & 2 & 3 & 6 \\
Pneumonia & 2 & 2 & 4 \\
Evisceração & 0 & 3 & 3 \\
Óbito & 0 & 2 & 2 \\
\hline
\end{tabular}

Em 47 pacientes $(57,3 \%)$ foram realizados exclusivamente procedimentos ginecológicos e em 35 casos $(42,7 \%)$ realizaram-se procedimentos não ginecológicos. Os procedimentos não ginecológicos incluíram: 17 colostomias, 16 enterectomias, 8 peritonectomias pélvicas, 7 colectomias, 5 ressecções parciais de cúpula frênica, 4 cistectomias parciais, 4 esplenectomias, 2 ileostomias e 1 hepatectomia parcial.

Como ilustra a Figura 1, todas as pacientes submetidas a procedimentos não ginecológicos apresentavam estadiamentos III e IV, ao passo que apenas $34,3 \%$ das pacientes submetidas a procedimentos ginecológicos apresentavam estadiamentos III e IV $(\mathrm{p}<0,001)$.

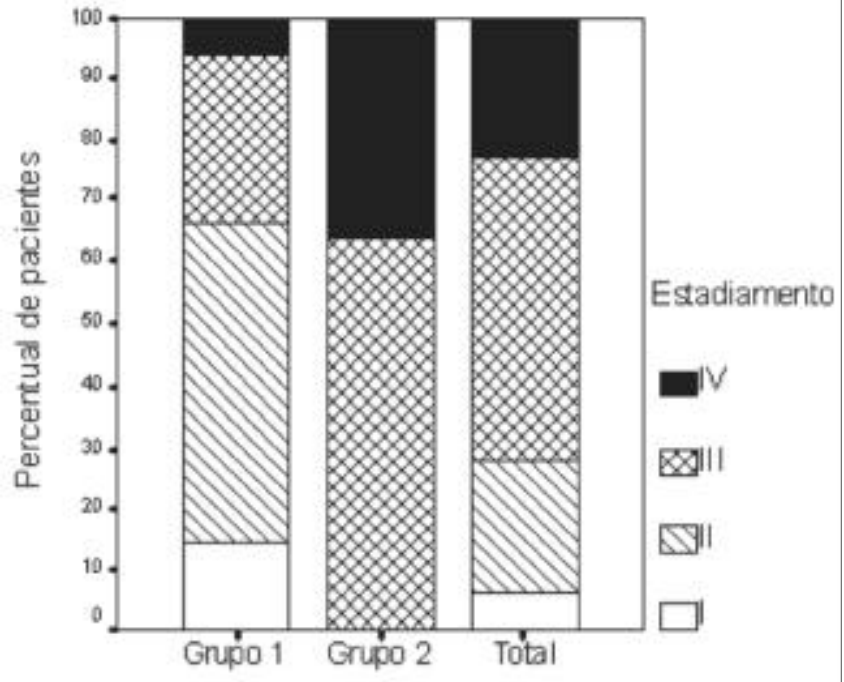

Figura 1 - Estadiamento cirúrgico do câncer de ovário (FIGO) nas pacientes submetidas a procedimentos cirúrgicos ginecológicos (grupo 1), procedimentos cirúrgicos não ginecológicos (grupo 2) e na totalidade dos casos (total).

O tempo cirúrgico médio foi superior no grupo de pacientes submetidas a procedimentos não ginecológicos $(5,3 \pm 1,4$ versus $3,1 \pm 0,9 ; p<0,001)$. Os grupos não apresentaram diferenças significativas em relação à necessidade de hemotransfusão $(42,2$ versus $40 \% ; p=0,512)$. A realização de procedimentos cirúrgicos não ginecológicos associou-se a maior taxa de complicações pós-operatórias (37 versus $17,1 \% ; \mathrm{p}=0,042$ ) (Tabela 2). As duas pacientes que evoluíram para o óbito no pósoperatório pertenciam ao grupo dos procedimentos cirúrgicos não ginecológicos (Tabela 1). Os grupos não apresentaram diferenças estatisticamente significativas em relação ao tempo de internação médio $(11,5 \pm 7,2$ versus $10 \pm 9,9$ dias; $\mathrm{p}=0,454)$ (Tabela 2).

Tabela 2 - Avaliação dos tempos médios de cirurgia, necessidade de hemotransfusão, presença de complicações pós-operatórias e tempo de internação médio nas pacientes submetidas a procedimentos cirúrgicos ginecológicos e naquelas submetidas a procedimentos cirúrgicos não ginecológicos.

\begin{tabular}{lccc}
\hline & $\begin{array}{l}\text { Procedimentos } \\
\text { ginecológicos }\end{array}$ & $\begin{array}{c}\text { Procedimentos } \\
\text { não ginecológicos }\end{array}$ & p \\
\hline $\begin{array}{l}\text { Tempo cirúrgico médio } \\
\text { (horas) }\end{array}$ & $3,1 \pm 0,9$ & $5,3 \pm 1,4$ & $<0,001$ \\
$\begin{array}{l}\text { Necessidade de } \\
\text { hemotransfusão }\end{array}$ & $14(40 \%)$ & $19(42,2 \%)$ & 0,512 \\
$\begin{array}{l}\text { Complicações } \\
\text { pós-operatórias }\end{array}$ & $6(17,1 \%)$ & $17(37 \%)$ & 0,042 \\
$\begin{array}{l}\text { Tempo de internação } \\
\text { (dias) }\end{array}$ & $10 \pm 9,9$ & $11,5 \pm 7,2$ & 0,454 \\
\hline As & & & \\
\hline
\end{tabular}

As comparações entre as médias dos grupos foram feitas pelo teste $t$ de Student. As comparações entre grupos independentes foram feitas pelo teste do $\chi^{2}$. As diferenças ou correlações com valor de $p<0,05$ foram consideradas significativas. 


\section{Discussão}

Esse estudo avaliou a utilização de procedimentos cirúrgicos não ginecológicos no tratamento do câncer de ovário em um centro terciário de tratamento com uma alta incidência de pacientes apresentando doença avançada ao diagnóstico. Para a obtenção de citorredução satisfatória foram utilizados procedimentos cirúrgicos não ginecológicos em $42,7 \%$ das pacientes. Isso pode ser justificado pelo estadiamento das pacientes operadas, pois $72 \%$ das pacientes apresentavam-se nos estádios III e IV, e pelo fato de que $37,2 \%$ das pacientes já haviam sido submetidas à cirurgia e/ou quimioterapia prévias.

A maior complexidade dos procedimentos cirúrgicos não ginecológicos aumentou o tempo operatório e a incidência de complicações pós-operatórias. Estudos avaliando pacientes submetidas a procedimentos cirúrgicos não ginecológicos em câncer de ovário mostram taxas de complicações de 29,8 a $59 \%$ e mortalidade pós-operatória de até $6 \%$ (Tabela 3).

Tabela 3 - Estudos nos quais se avaliaram a morbidade e mortalidade da realização de procedimentos cirúrgicos não ginecológicos no tratamento do câncer de ovário.

\begin{tabular}{|c|c|c|c|c|c|c|}
\hline Autor & $\begin{array}{c}\mathrm{N}^{0} \\
\text { pacientes }\end{array}$ & $\begin{array}{l}\text { Transfusão } \\
\text { (unidades) }\end{array}$ & $\begin{array}{l}\text { Tempo cirúrgico } \\
\text { (horas) }\end{array}$ & $\begin{array}{c}\text { Complicações } \\
\text { PO(\%) }\end{array}$ & Dias de internação & Mortalidade(\%) \\
\hline Berek et al., $1984^{21}$ & 72 & 1,28 & 3,5 & Não avaliado & Não avaliado & 0 \\
\hline Eisenkop et al., $1992^{22}$ & 163 & 1,19 & 4,2 & 41,7 & 12 & 1.8 \\
\hline Tamussino et al., $2001^{15}$ & 364 & Não avaliado & 3,4 & 29,8 & 11 & 3,9 \\
\hline Gillette-Cloven et al., $2001^{11}$ & 104 & 1,44 & 4,5 & 59 & 16 & 6 \\
\hline \multicolumn{7}{|l|}{ Silva-Filho et al., $2004^{*}$} \\
\hline (presente estudo) & 37 & Não avaliado & 5,6 & 37,8 & 12 & 5,7 \\
\hline
\end{tabular}

*Incluídas somente as pacientes submetidas a procedimentos cirúrgicos não ginecológicos.

A maior parte dos procedimentos não ginecológicos dessa casuística envolveu o trato gastrintestinal, especialmente a realização de colostomias e enterectomias. Um trabalho, com 364 pacientes com câncer de ovário, mostrou que as cirurgias gastrintestinais são freqüentes, tendo a citorredução como principal indicação, seguida pelos quadros de obstrução intestinal ${ }^{15}$. A exenteração pélvica posterior foi descrita, em pacientes nos estádios III e IV, como alternativa para a obtenção de uma citorredução satisfatória ${ }^{15}$. Um estudo retrospectivo, com 105 pacientes portadoras de câncer de ovário nos estádios III e IV, mostrou a citorredução primária como causa de $92 \%$ das cirurgias sobre o trato gastrintestinal ${ }^{17}$.

Foram realizadas quatro esplenectomias devido à presença de implantes tumorais no hilo esplênico ( 3 casos) ou metástases parenquimatosas (1 caso). A indicação de esplenectomia para se obter uma citorredução satisfatória é controversa. Tal procedimento foi associado a uma alta morbidade pós-operatória em pacientes com câncer de ovário ${ }^{18}$. As complicações relacionadas a esplenectomia incluem lesão pancreática, pesudocisto de pâncreas, derrame pleural e atelectasia ${ }^{19}$. Um estudo ava- liou 258 pacientes com câncer de ovário nas quais foram realizadas 13 esplenectomias. Esse procedimento foi considerado adequado e seguro, porém indicado apenas naquelas pacientes com possibilidade de citorredução ótima ${ }^{20}$.

A peritonectomia pélvica foi realizada em 8 pacientes $(9,5 \%)$. A ressecção em bloco dos ovários, útero e peritônio pélvico constitui uma alternativa cirúrgica para a obtenção de citorredução efetiva $^{21,22}$. Nos casos de carcinomatose peritoneal, a peritonectomia associada à quimioterapia intraperitoneal possibilita alta concentração local de drogas citostáticas ${ }^{23}$.

Foi demonstrado que a sobrevida de pacientes com câncer de ovário operadas por um oncologista ginecológico é superior à daquelas operadas por outro especialista ${ }^{22}$. O presente estudo mostrou a alta freqüência na realização de procedimentos não ginecológicos no tratamento do câncer de ovário, sendo imprescindivel ao oncologista ginecológico a realização de cirurgias gastrintestinais, proctológicas e urológicas. O domínio dessas cirurgias pode alterar, inclusive, os critérios de ressecabilidade dos tumores, propiciando à paciente maior chance de citorredução satisfatória. 
Concluímos que os procedimentos cirúrgicos não ginecológicos são freqüentemente empregados no tratamento de pacientes com câncer de ovário. A realização dessas cirurgias está associada a maior tempo cirúrgico e maior taxa de complicações pós-operatórias.

\section{ABSTRACT}

Objective: to evaluate the incidence of non-gynecological surgical procedures used in the treatment of ovarian cancer, as well to describe their complications.

Methods: eighty-two patients with ages from 22 to 89 (mean $=54.1 \pm 15.1$ years), submitted to laparotomy for surgical treatment of ovarian cancer from February 1999 to October 2003 were retrospectively evaluated. This study included only patients with epithelial ovary carcinoma. The patients were divided into 2 groups, patients submitted exclusively to gynecological procedures and patients submitted to non-gynecological procedures. Statistical analysis was made with the Student's t-test or the chi-square test.

Results: 5 patients (6.1\%) were in stage (FIGO) I, 18 (21.9\%) in stage II, $40(48.8 \%)$ in stage III, and 19 $(23.2 \%)$ in stage IV. Non-gynecological procedures were done in 35 cases (42.7\%), including: 17 colostomies, 16 enterectomies, 8 peritonectomies, 7 colectomies, 5 partial diaphragm resections, 4 partial cystectomies, 4 splenectomies, 2 ileostomies, and 1 hepatectomy. All patients submitted to non-gynecological procedures were included in stages III and IV. This group of patients underwent longer-lasting surgeries $(5.3 \pm 1.4$ versus 3.1 $+0,0 \mathrm{~h} ; \mathrm{p}<0.001)$. There was no significant difference between these two groups regarding hemotransfusion requirement $(42,2$ versus $40 \% ; p=0.512)$ and hospitalization time $(11.5 \pm 7.2$ versus $10 \pm 9.9$ days; $p=$ $0.454)$. Patients submitted to non-gynecological surgeries developed higher rates of postoperative complications ( 37 versus $17.1 \% ; p=0.042)$, and two of them (2.4\%) died.

Conclusions: non-gynecological surgical procedures are frequently used in the treatment of patients with ovarian cancer. These procedures are associated with a longer-lasting surgery and higher rates of postoperative complications.

KEYWORDS: Ovarian cancer. Cytoreduction. Surgical complications.

\section{Referências}

1. Landis SH, Murray T, Bolden S, Wingo PA. Cancer statistics, 1999. CA Ca-A Cancer J Clin 1999; 49: $8-31$

2. Estimativa da incidência e mortalidade por câncer no Brasil 1999. Ministério da Saúde INCA / Conprev. Rio de Janeiro, 1999. INCA.gov.br

3. Pecorelli S, Benedet JL, Creasman WT, et al. Annual report on the results of treatment in gynecological cancer. Oxford: International Federation of Gynecology and Obstetrics; 1998.

4. Ries LA, Kosary CL, Hankey BF, et al. SEER Cancer statistics review 1973-1995. Bethesda: National Cancer Institute; 1998.

5. Hillner BE, Smith TJ, Desch CE. Hospital and physician volume or specialization and outcomes in cancer treatment: importance in quality of cancer care. J Clin Oncol 2000; 18:2327-40.

6. Meigs JV. Tumors of the Female Pelvic Organs $1^{\text {st }}$ ed. New York: Macmillan, 1934.

7. Munnell EW. The changing prognosis and treatment in cancer of the ovary: a report of 235 patients with primary ovarian carcinoma. Am J Obstet Gynecol 1968; 100: 790-805.

8. Ball H, Berek JS. The effect of diameter of largest residual disease on survival after primary cytoreductive surgery in patients with suboptimal residual epithelial ovarian carcinoma. Am J Obstet Gynecol 1994;170: 974-80.

9. Griffiths CT. Surgical resection of tumor bulk in the primary treatment of ovarian carcinoma. Natl Cancer Inst Monogr 1975; 42:101-4.

10.Hoskins WJ, Bundy BN, Thigpen JT, Omura GA. The influence of cytoreductive surgery on recurrencefree interval and survival in small-volume stage III epithelial ovarian cancer: a Gynecologic Oncology Group study. Gynecol Oncol 1992; 47:159-60.

11.Gillette-Cloven N, Burger RA, Monk BJ, et al. Bowel resection during primary cytoreduction. J Am Coll Surg 2001; 193:626-32.

12.Bristow RE, Tomacruz RS, Armstrong DK, Trimble EL, Montz FJ. Survival effect of maximal cytoreductive surgery for advanced ovarian carcinoma during the platinum era: a meta-analysis. J Clin Oncol 2002; 20:1248-59.

13. Pickel H, Burghardt E, Webb MJ, et al. Spread of ovarian cancer. In: Burghardt, E, editor. Surgical Gynecologic Oncology. $1^{\text {st }}$ ed. Stuttgart: Thieme; 1993. p. 435-9.

14.Rutledge FN. The gynecologic oncologist. His responsibility and training. Obstet Gynecol 1972; 40:749-54. 
15.Tamussino KF, Lim PC, Webb MJ, Lee RA, Lesnick TG. Gastrointestinal surgery in patients with ovarian cancer. Gynecol Oncol 2001; 80:79-84.

16.Odicino F, Favalli G, Zigliani L, Pecorelli S. Staging of gynecologic malignancies. Surg Clin North Am 2001; 81:753-70.

17.Eisenkop SM, Nalick RH, Teng NN. Modified posterior exenteration for ovarian cancer. Obstet Gynecol 1991; 78:879-85.

18. Morris M, Gershenson DM, Burke TW, Wharton JT, Copeland LJ, Rutledge FN. Splenectomy in gynecologic oncology: indications, complications, and technique. Gynecol Oncol 1991; 43:119-22.

19.Nicklin JL, Copeland LJ, O’Toole RV, Lewandowski GS, Vaccarello L, Havenar LP. Splenectomy as part of cytoreductive surgery for ovarian carcinoma. Gynecol Oncol 1995; 58:244-7.
20.Bilgin T, Ozerkan K, Ozan H. Splenectomy in cytoreductive surgery for advanced ovarian cancer. Arch Gynecol Obstet. In press, February 2004.

21.Berek JS, Hacker NF, Lagasse LD. Rectosigmoid colectomy and reanastomosis to facilitate resection of primary and recurrent gynecologic cancer. Obstet Gynecol 1984; 64:715-20.

22.Eisenkop SM, Spirtos NM, Montag TW, Nalick RH, Wang HJ. The impact of subspecialty training on the management of advanced ovarian cancer. Gynecol Oncol 1992; 47:203-9.

23.Sugarbaker PH. Successful management of microscopic residual disease in large bowel cancer. Cancer Chemother Pharmacol 1999; 43:Suppl: S15-25.

Recebido em: 5/2/2004 Aceito com modificações em: 3/5/2004 